\title{
THE BRITISH ASSET-OR-NOTHING PUT OPTION
}

DOI:

10.1142/S0219024917500303

\section{Document Version}

Accepted author manuscript

Link to publication record in Manchester Research Explorer

\section{Citation for published version (APA):}

GAO, MIN. (2017). THE BRITISH ASSET-OR-NOTHING PUT OPTION. International Journal of Theoretical and Applied Finance, 20. https://doi.org/10.1142/S0219024917500303

\section{Published in:}

International Journal of Theoretical and Applied Finance

\section{Citing this paper}

Please note that where the full-text provided on Manchester Research Explorer is the Author Accepted Manuscript or Proof version this may differ from the final Published version. If citing, it is advised that you check and use the publisher's definitive version.

\section{General rights}

Copyright and moral rights for the publications made accessible in the Research Explorer are retained by the authors and/or other copyright owners and it is a condition of accessing publications that users recognise and abide by the legal requirements associated with these rights.

\section{Takedown policy}

If you believe that this document breaches copyright please refer to the University of Manchester's Takedown Procedures [http://man.ac.uk/04Y6Bo] or contact uml.scholarlycommunications@manchester.ac.uk providing relevant details, so we can investigate your claim.

\section{OPEN ACCESS}




\title{
The British Asset-or-Nothing Put Option
}

\author{
Min Gao
}

\begin{abstract}
Following the economic rationale of Peskir and Samee, we present a new class of asset-or-nothing put option where the holder enjoys the early exercise feature of American asset-or-nothing put option whereupon his payoff is the 'best prediction' of the European asset-or-nothing put option payoff under the hypothesis that the true drift equals a contract drift. Based on the observed price movements, the option holder finds that the true drift of the stock price is unfavourable then he can substitute it with the contract drift and minimise his losses. The key to the British asset-or-nothing put option is the protection feature as not only can the option holder exercise at or above the strike price to a substantial reimbursement of the original option price (covering the ability to sell in a liquid option market completely endogenously) but also when the stock price movements are favourable he will generally receive high returns. We derive a closed form expression for the arbitrage-free price in terms of the rational exercise boundary and show that the rational exercise boundary itself can be characterised as the unique solution to a non-linear integral equation. We also analyse the financial meaning of the British asset-or-nothing put option using the results above and show that with the contract drift properly selected the British asset-or-nothing put option becomes a very attractive alternative to the classic European/American asset-or-nothing put option.
\end{abstract}

\section{Introduction}

The purpose of the present paper is to introduce and examine the British payoff mechanism by Peskir and Samee $(2011,2013)$ in the context of asset-or-nothing put option that is one type of the binary options. There are various types of binary options that may be considered to this end: (i) calls and puts; (ii) cash-or-nothing and asset-or-nothing. This leads to an extensive programme of research that we open in this paper by focusing on the asset-or-nothing put option as we believe that this is the most interesting case from a mathematical point of view.

Following the economic rationale of the British put and call options, we introduce a new asset-or-nothing put option that endogenously provides its holder with a protection mechanism against unfavourable stock price movements. This mechanism is intrinsically built into the option contract using the concept of optimal prediction (Du Toit and Peskir, 2007). We refer

Key words and phrases: British asset-or-nothing put option, American asset-or-nothing put option, arbitrage-free price, rational exercise boundary, optimal stopping, geometric Brownian motion, parabolic free boundary problem. 
to such contracts as 'British' for the reasons outlined by Peskir and Samee (2011, 2013). Similar to the British put and call options, the British asset-or-nothing put option not only provides a unique protection against unfavourable stock price movements (see more details in section 5) but also enables the option holder to obtain higher returns when the stock price movements are favourable in both liquid and illiquid markets. This reaffirms the fact noted by Al-Fagih (2014, 2015), Glover et al. (2010, 2011) and Kitapbayev (2015) for the value of British barrier, British Asian, British Russian and British lookback options that the British feature of optimal prediction can provide both protection against unfavourable price movements as well as securing high returns when movements are favourable. These combined features are especially appealing as the problems of liquidity and return are addressed completely endogenously.

The paper mainly consists of two parts: analytical solution and financial analysis. According to the financial theory (Shiryaev and Kruzhilin, 1999), the arbitrage-free price of the option is a solution to an optimal stopping problem with the gain function as the payoff of the option. Following the notion 'compound option' by Geske (1979) where an analytical solution for compound option was derived, we refer to a more recent paper by Gukhal (2004) for an informative review. We then use a local time-space calculus on curves (Peskir, 2005) to derive a closed form expression for the arbitrage-free price in terms of the optimal stopping boundary and show that the free boundary itself is the unique solution to a non-linear integral equation. We perform the analysis of returns of the British asset-or-nothing put option with the American asset-or-nothing put option. After observing the returns upon exercising, we conclude the remarkable protection feature of the British asset-or-nothing put option.

The paper is organised as follows. In Section 2 we present a basic motivation for the British asset-or-nothing put option. In Section 3 we firstly formally define the British asset-or-nothing put option and present some of its basic properties. We continue in Section 4 to derive a closed form expression for the arbitrage-free price in terms of the rational exercise boundary and show that the rational exercise boundary can be characterised as the unique solution to a non-linear integral equation. In Section 5 we provide a financial analysis using the results above, making a comparison with American asset-or-nothing put option.

\section{Basic motivation for the British asset-or-nothing put option}

The basic economic motivation for the British asset-or-nothing put option is parallel to that of British put and call options (Peskir and Samee, 2011, 2013). In this section, we briefly review the key elements of the motivation on the asset-or-nothing put option.

1. Consider the financial market consisting a risky stock $X$ and a riskless bond $B$ whose prices respectively evolve as

$$
\begin{aligned}
& d X_{t}=\mu X_{t} d t+\sigma X_{t} d W_{t} \\
& d B_{t}=r B_{t} d t
\end{aligned}
$$

with $X_{0}=x$ and $B_{0}=1$ where $\mu \in \mathrm{R}$ is the drift, $\sigma>0$ is the volatility coefficient, $W=\left(W_{t}\right)_{t \geq 0}$ is a standard Brownian motion defined on a probability space $(\Omega, \mathcal{F}, \mathrm{P})$ and $r>0$ is the interest rate. Recall that the European (American) asset-or-nothing put option (Black and Scholes, 
1973) is a financial contract between a seller/hedger and a buyer/holder entitling the latter to receive the payoff of the underlying asset $X_{t}$ at specified maturity time $T>0$ in the European case (or any stopping time prior to $T$ in the American case). Standard hedging arguments based on self-financing portfolios imply that the arbitrage-free price of the option is given by (Merton, 1973)

$$
\begin{aligned}
& V=\mathrm{E}^{\mathrm{Q}}\left[e^{-r T} X_{T} \mathrm{I}\left(X_{T} \leq K\right)\right] \quad \text { (European asset-or-nothing put option) } \\
& V=\sup _{0 \leq \tau \leq T} \mathrm{E}^{\mathrm{Q}}\left[e^{-r \tau} X_{\tau} \mathrm{I}\left(X_{\tau} \leq K\right)\right] \quad \text { (American asset-or-nothing put option) }
\end{aligned}
$$

where the expectation $E^{Q}$ is taken with respect to the unique equivalent martingale measure Q (Shiryaev and Kruzhilin, 1999) and the supremum is taken over all stopping times $\tau$ of $X$ with values in $[0, T]$. In this section we will analyse the option from the standpoint of a true buyer (Peskir and Samee, 2011, 2013). By 'true buyer' we mean a buyer who has no ability or desire to sell the option nor to hedge his own position. Thus every true buyer will exercise the option in accordance with the rational performance (at time $T$ in the European case or at the optimal stopping time $\tau_{*}$ in the American case). More details on the motivation and a true buyer are given by Peskir and Samee (2011).

2. With this in mind we now return to the asset-or-nothing put option holder whose payoff is given by

$$
e^{-r T} X_{T}(\mu) \mathrm{I}\left(X_{T}(\mu) \leq K\right)
$$

where $X_{T}=X_{T}(\mu)$ represents the stock price at time $T$ under the actual probability measure $P$. Recall that the unique strong solution to (2.1) is given by

$$
X_{t}(\mu)=x \exp \left(\sigma W_{t}+\left(\mu-\sigma^{2} / 2\right) t\right)
$$

under $\mathrm{P}$ for $t \in[0, T]$ and $\mu \in \mathrm{R}$ is the real drift. Inserting (2.6) into (2.5) we find that the expected value of the buyer's payoff equals

$$
P=P(\mu)=\mathrm{E}\left[e^{-r T} X_{T} \mathrm{I}\left(X_{T} \leq K\right)\right]=x e^{(\mu-r) T} \mathrm{P}\left(X_{T}(\mu) \leq K\right)
$$

Moreover, it is well known that $\operatorname{Law}(X(\mu) \mid \mathrm{Q})=\operatorname{Law}(X(r) \mid \mathrm{P})$ so that the arbitrage-free price of the option equals

$$
V=P(r)=x \mathrm{P}\left(X_{T}(r) \leq K\right)
$$

A comparison of (2.7) and (2.8) shows that if $\mu=r$ then the return is 'fair' for the buyer, in the sense that $V=P$, where $V$ represents the value of his investment and $P$ represents the expected value of his payoff. On the other hand, if $\mu<r$ then the return is 'favourable' for the buyer, in the sense that $V<P$, and if $\mu>r$ then the return is 'unfavourable' for the buyer, in the sense that $V>P$ with the same interpretations above. In the real financial market, the actual drift $\mu$ is unknown at time $t=0$ and is not easy to estimate at later times $t \in(0, T]$ in finite horizon.

3. The brief analysis above shows that one will buy the asset-or-nothing put option if he believes that $\mu<r$ and surely he is pleased to see what reaffirm his belief. The British asset-ornothing put option aims to address the opposite situation: what if the option holder observes 
stock price movements that change his belief regarding the actual drift and cause him to believe that $\mu>r$ instead? In this contingency the British asset-or-nothing option holder is effectively able to substitute this unfavourable drift with a contract drift and minimise his losses. In this way he is endogenously protected from any stock price drift greater than the contract drift. The value of the contract drift is therefore selected to represent the buyer's expected level of tolerance for the deviation of the actual drift from his original belief. It will be shown below (similar to Peskir and Samee $(2011,2013)$ ) that the practical implications of this protection feature are most remarkable as not only is the option holder afforded a unique protection against unfavourable stock price movements but also when the stock price movements are favourable he will generally receive high returns. Further comments regarding the option holder's ability to sell his contract are shown in the final paragraph of Section 2 by Peskir and Samee (2011).

\section{The British asset-or-nothing put option: Definition and basic properties}

We begin this section from a formal definition of the British asset-or-nothing put option and then briefly analyse the free-boundary problem.

Definition 1. The British asset-or-nothing put option is a financial contract between a seller/ hedger and a buyer/holder entitling the latter to exercise at any (stopping) time $\tau$ prior to $T$ whereupon his payoff is the 'best prediction' of the European asset-or-nothing put option payoff $X_{T} \mathrm{I}\left(X_{T} \leq K\right)$ given all the information up to time $\tau$ under the hypothesis that the true drift of the stock price equals $\mu_{c}$.

Note that $\mu_{c}$ stands for the buyer's level of tolerance for the deviation of the true drift $\mu$ from his original belief. The contract drift $\mu_{c}$ satisfies

$$
\mu_{c}>r \quad \text { or } \quad \mu_{c}<0
$$

If (3.1) does not hold, the buyer would be overprotected and exercise the option immediately (more details will be given below).

1. We define the payoff function $G^{\mu_{c}}$ as

$$
G^{\mu_{c}}\left(t, X_{t}\right)=\mathrm{E}^{\mu_{c}}\left[X_{T} \mathrm{I}\left(X_{T} \leq K\right) \mid \mathcal{F}_{t}\right]
$$

Then it is followed that $G^{\mu_{c}}(t, x)$ can be written as

$$
G^{\mu_{c}}(t, x)=\mathrm{E}\left[x Z_{T-t}^{\mu_{c}} \mathrm{I}\left(x Z_{T-t}^{\mu_{c}} \leq K\right)\right]
$$

and $Z_{T-t}^{\mu_{c}}$ is given by

$$
Z_{T-t}^{\mu_{c}}=\exp \left(\sigma W_{T-t}+\left(\mu_{c}-\sigma^{2} / 2\right)(T-t)\right)
$$

for $t \in[0, T]$ and $x \in(0, \infty)$. Inserting (3.4) into (3.3), we get

$$
\begin{aligned}
G^{\mu_{c}}(t, x) & =\mathrm{E}\left[x Z_{T-t}^{\mu_{c}} \mathrm{I}\left(x Z_{T-t}^{\mu_{c}} \leq K\right)\right] \\
& =x e^{\mu_{c}(T-t)} \Phi\left(\frac{1}{\sigma \sqrt{T-t}}\left[\ln \frac{K}{x}-\left(\mu_{c}-\frac{1}{2} \sigma^{2}\right)(T-t)\right]\right)
\end{aligned}
$$


where $\Phi$ is the standard normal distribution function.

2. The arbitrage-free price of the British asset-or-nothing put option is given by

$$
V=\sup _{0 \leq \tau \leq T} \mathrm{E}^{\mathrm{Q}}\left[e^{-r \tau} \mathrm{E}^{\mu_{c}}\left[X_{T} \mathrm{I}\left(X_{T} \leq K\right) \mid \mathcal{F}_{\tau}\right]\right]
$$

where the supremum is taken over all stopping times $\tau$ of $X$ with values in $[0, T]$. Similarly, the expression (3.6) extends to

$$
V(t, x)=\sup _{0 \leq \tau \leq T-t} \mathrm{E}_{t, x}^{\mathrm{Q}}\left[e^{-r \tau} G^{\mu_{c}}\left(t+\tau, X_{t+\tau}\right)\right]
$$

where the supremum is taken over all stopping times $\tau$ of $X$ with values in $[0, T-t]$ and $\mathrm{E}_{t, x}^{\mathrm{Q}}$ is taken with respect to the (unique) equivalent martingale measure $\mathrm{Q}$ under which $X_{t}=x$. It follows that

$$
V(t, x)=\sup _{0 \leq \tau \leq T-t} \mathrm{E}_{t, x}\left[e^{-r \tau} G^{\mu_{c}}\left(t+\tau, x X_{\tau}\right)\right]
$$

for $t \in[0, T]$ and $x \in(0, \infty)$ where the supremum is taken as in $(3.7)$ and the process $X=X(r)$ under $\mathrm{P}$ solves

$$
d X_{t}=r X_{t} d t+\sigma X_{t} d W_{t}
$$

with $X_{0}=1$.

3. To get the solution to the optimal stopping problem (3.8), apply Ito's formula and get

$$
e^{-r s} G^{\mu_{c}}\left(t+s, X_{t+s}\right)=G^{\mu_{c}}(t, x)+\int_{0}^{s} e^{-r u} H^{\mu_{c}}\left(t+u, X_{t+u}\right) d u+M_{s}
$$

where the function $H^{\mu_{c}}=H^{\mu_{c}}(t, x)$ is defined by

$$
H^{\mu_{c}}=G_{t}^{\mu_{c}}+r x G_{x}^{\mu_{c}}+\frac{\sigma^{2}}{2} x^{2} G_{x x}^{\mu_{c}}-r G^{\mu_{c}}
$$

and $M_{s}=\sigma \int_{0}^{s} e^{-r u} X_{u} G_{x}^{\mu_{c}}\left(t+u, X_{t+u}\right) d W_{u}$ is a continuous martingale for $s \in[0, T-t]$ with $t \in[0, T)$. From the optional sampling theorem we get

$$
\mathrm{E}\left[e^{-r \tau} G^{\mu_{c}}\left(t+\tau, X_{t+\tau}\right)\right]=G^{\mu_{c}}(t, x)+\mathrm{E}\left[\int_{0}^{\tau} e^{-r u} H^{\mu_{c}}\left(t+u, x X_{u}\right) d u\right]
$$

for all stopping times $\tau$ of $X$ with values in $[0, T-t]$ with $t \in[0, T)$ and $x \in(0, \infty)$ given and fixed. Moreover, the payoff function satisfies the Kolmogorov backward equation (Peskir and Shiryaev, 2006)

$$
G_{t}^{\mu_{c}}+\mu_{c} x G_{x}^{\mu_{c}}+\frac{\sigma^{2}}{2} x^{2} G_{x x}^{\mu_{c}}=0
$$

thus we get

$$
H^{\mu_{c}}=\left(r-\mu_{c}\right) x G_{x}^{\mu_{c}}-r G^{\mu_{c}}
$$




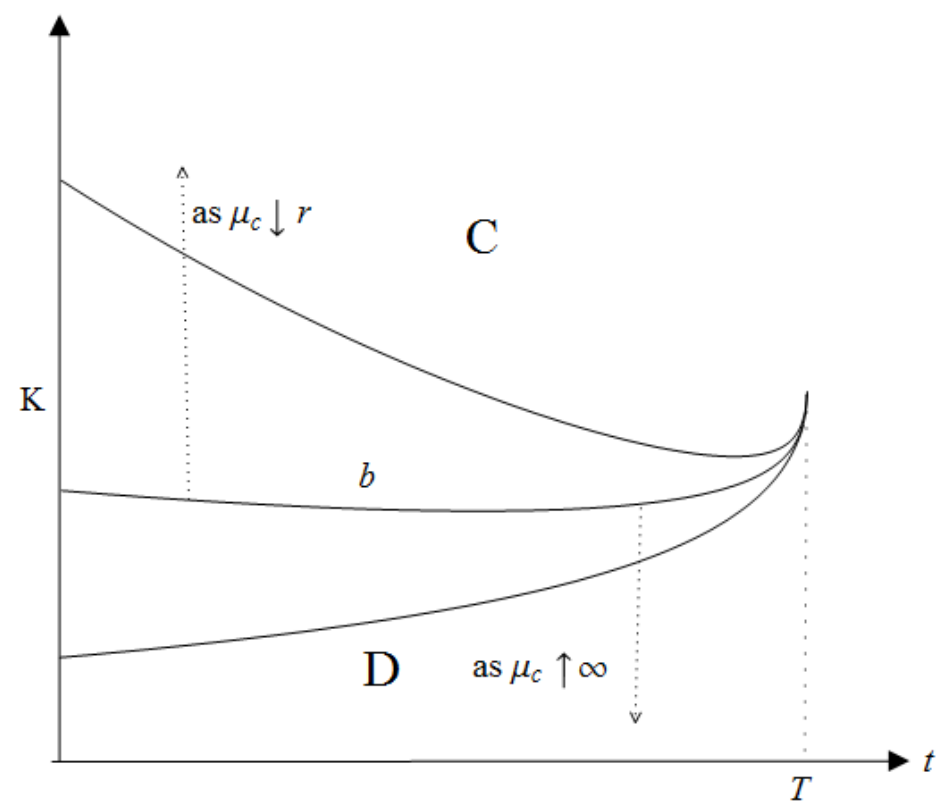

Figure 1: A computer drawing showing how the rational exercise boundary of the British asset-or-nothing put option changes as one varies the contract drift with $\mu_{c}>r$.

It is easy to calculate $G_{x}^{\mu_{c}}$ from (3.5) and substituting $G_{x}^{\mu_{c}}$ and $G^{\mu_{c}}$ into (3.14), we get

$$
\begin{aligned}
H^{\mu_{c}}(t, x)= & \left(\mu_{c}-r\right) e^{\mu_{c}(T-t)} \frac{x}{\sigma \sqrt{T-t}} \varphi\left(\frac{1}{\sigma \sqrt{T-t}}\left[\ln \frac{K}{x}-\left(\mu_{c}-\frac{1}{2} \sigma^{2}\right)(T-t)\right]\right) \\
& -\mu_{c} x e^{\mu_{c}(T-t)} \Phi\left(\frac{1}{\sigma \sqrt{T-t}}\left[\ln \frac{K}{x}-\left(\mu_{c}-\frac{1}{2} \sigma^{2}\right)(T-t)\right]\right)
\end{aligned}
$$

for $t \in[0, T)$ and $x \in(0, \infty)$ and $\varphi(y)=\frac{1}{\sqrt{2 \pi}} e^{-y^{2} / 2}$ for $y \in \mathrm{R}$.

On closer examination of the function $H^{\mu_{c}}$ in (3.15) and we find using elementary arguments that there exists a $C^{1}$ function $h:[0, T] \rightarrow \mathrm{R}$ such that

$$
H^{\mu_{c}}(t, h(t))=0
$$

for all $t \in[0, T)$. Define the set

$$
\left\{H^{\mu_{c}}<0\right\}:=\left\{(t, x) \in[0, T] \times(0, \infty): H^{\mu_{c}} \leq 0\right\}
$$

We distinguish two functions $h_{1}$ and $h_{2}$ satisfying (3.16) such that (i) $\left\{H^{\mu_{c}} \leq 0\right\}=\{(t, x) \in$ $\left.[0, T) \times(0, \infty): x \leq h_{1}(t)\right\}$ when $\mu_{c}>r$ and (ii) $\left\{H^{\mu_{c}} \leq 0\right\}=\{(t, x) \in[0, T) \times(0, \infty):$ $\left.x \geq h_{2}(t)\right\}$ when $\mu_{c}<0$. Moreover, it can be verified that if $(t, x) \in\left\{H_{\mu_{c}} \leq 0\right\}$ and $t<T$ is sufficiently close to $T$ then it is optimal to stop immediately (since the gain obtained from being outside this set cannot offset the cost of getting there due to the lack of time). This shows that the optimal stopping boundary $b:[0, T] \rightarrow[0, \infty]$ separating the continuation set from the stopping set satisfies $b(T)=h(T)$ and this value equals $K$.

4. Introducing the continuation set

$$
C=\left\{(t, x) \in[0, T) \times(0, \infty) \mid V(t, x)>G^{\mu_{c}}(t, x)\right\}
$$




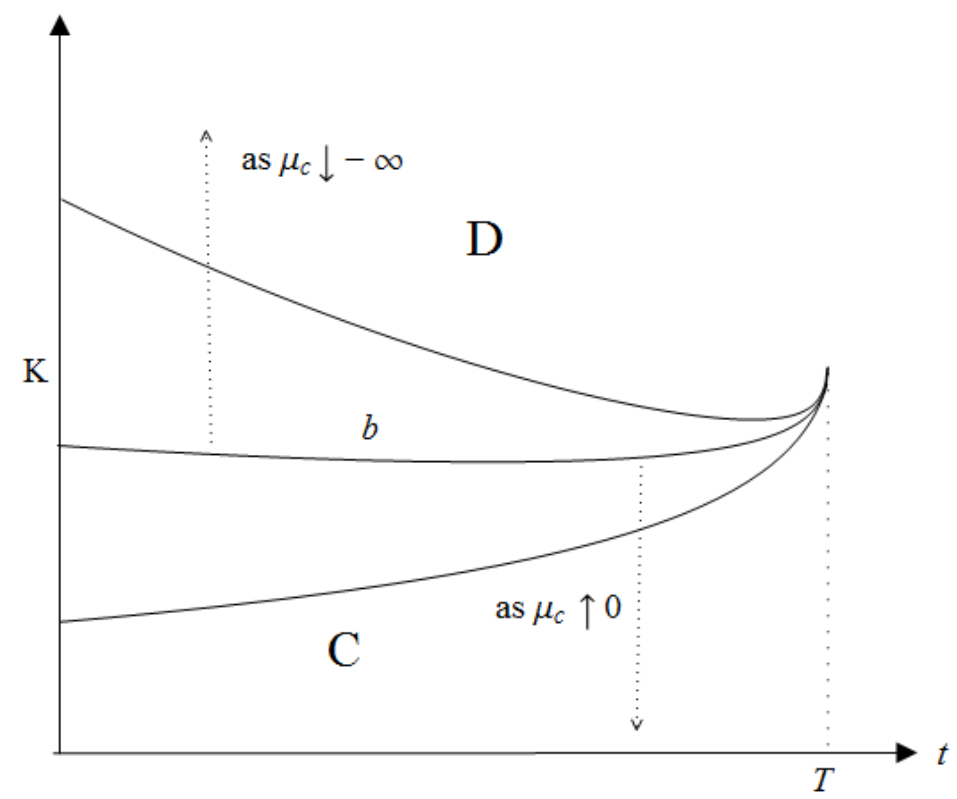

Figure 2: A computer drawing showing how the rational exercise boundary of the British asset-or-nothing put option changes as one varies the contract drift with $\mu_{c}<0$.

and the stopping set

$$
D=\left\{(t, x) \in[0, T) \times(0, \infty) \mid V(t, x)=G^{\mu_{c}}(t, x)\right\}
$$

we may infer from general theory of optimal stopping for Markov processes (Peskir and Shiryaev, 2006) that the optimal stopping time in (3.8) is given by

$$
\tau_{D}=\inf \left\{s \in[0, T-t]:\left(t+s, X_{t+s}\right) \in D\right\} .
$$

Remark 2. It is important to note that the proper selection of the contract drift $\mu_{c}$ leads to different continuation set $C$ and different stopping set $D$. If $\mu_{c}>r$, the optimal stopping time in the problem (3.8) is given by (see Figure 1)

$$
\tau_{b_{1}}=\inf \left\{t \in[0, T] \mid X_{t} \leq b_{1}(t)\right\}
$$

where the optimal stopping boundary $b_{1}=b_{1}(t)$ is to be determined. On the other hand, when $\mu_{c}<0$ the optimal stopping time is given by (see Figure 2)

$$
\tau_{b_{2}}=\inf \left\{t \in[0, T] \mid X_{t} \geq b_{2}(t)\right\}
$$

where the optimal stopping boundary $b_{2}=b_{2}(t)$ is to be determined.

(i) For $\mu_{c}>r$, standard Markovian arguments lead to the following free-boundary problem (Peskir and Shiryaev, 2006)

$$
V_{t}+r x V_{x}+\frac{\sigma^{2}}{2} x^{2} V_{x x}-r V=0 \quad \text { for } \quad x>b_{1}(t) \text { and } t \in[0, T)
$$




$$
\begin{aligned}
& V(t, x)=G^{\mu_{c}}(t, x) \text { for } x=b_{1}(t) \text { and } t \in[0, T) \\
& V_{x}(t, x)=G_{x}^{\mu_{c}}(t, x) \text { for } x=b_{1}(t) \text { and } t \in[0, T) \\
& V(T, x)=x \mathrm{I}(x \leq K) \text { for } x \geq b_{1}(T)=K \\
& V(t, \infty)=0 \text { for } t \in[0, T) .
\end{aligned}
$$

The continuation set can be expressed as

$$
C=\left\{V>G^{\mu_{c}}\right\}=\left\{(t, x) \in[0, T) \times(0, \infty) \mid x>b_{1}(t)\right\}
$$

and the stopping set is given by

$$
D=\left\{V=G^{\mu_{c}}\right\}=\left\{(t, x) \in[0, T) \times(0, \infty) \mid x \leq b_{1}(t)\right\} \cup\left\{(T, x) \mid x>b_{1}(T)\right\} .
$$

(ii) For $\mu_{c}<0$, similarly, we formulate the following free-boundary problem

$$
\begin{aligned}
& V_{t}+r x V_{x}+\frac{\sigma^{2}}{2} x^{2} V_{x x}-r V=0 \text { for } x<b_{2}(t) \text { and } t \in[0, T) \\
& V(t, x)=G^{\mu_{c}}(t, x) \text { for } x=b_{2}(t) \text { and } t \in[0, T) \\
& V_{x}(t, x)=G_{x}^{\mu_{c}}(t, x) \text { for } x=b_{2}(t) \text { and } t \in[0, T) \\
& V(T, x)=x \mathrm{I}(x \leq K) \text { for } x \geq b_{2}(T)=K \\
& V(t, \infty)=0 \text { for } t \in[0, T) .
\end{aligned}
$$

The continuation set can be expressed as

$$
C=\left\{V>G^{\mu_{c}}\right\}=\left\{(t, x) \in[0, T) \times(0, \infty) \mid x<b_{2}(t)\right\}
$$

and the stopping set is given by

$$
D=\left\{V=G^{\mu_{c}}\right\}=\left\{(t, x) \in[0, T) \times(0, \infty) \mid x \geq b_{2}(t)\right\} \cup\left\{(T, x) \mid x<b_{2}(T)\right\} .
$$

\section{The arbitrage-free price and the rational exercise bound- ary}

In this section we will derive a closed form expression for the arbitrage-free price $V$ in terms of the rational exercise boundary $b$ (the early-exercise premium representation) for the British asset-or-nothing put option.

We will make use of the following functions in Theorem 3 below

$$
\begin{aligned}
& F(t, x)=G^{\mu_{c}}(t, x)-e^{-r(T-t)} G^{r}(t, x) \\
& J_{1}(t, x, v, z)=-e^{-r(v-t)} \int_{0}^{z} H^{\mu_{c}}(v, y) f(v-t, x, y) d y \\
& J_{2}(t, x, v, z)=-e^{-r(v-t)} \int_{z}^{\infty} H^{\mu_{c}}(v, y) f(v-t, x, y) d y
\end{aligned}
$$


for $t \in[0, T), x>0, v \in(t, T)$ and $y>0$, where functions $G^{r}$ and $G^{\mu_{c}}$ are given in (3.3) above, the function $H^{\mu_{c}}$ is given in (3.15) above, and $y \mapsto f(v-t, x, y)$ is the probability density function of $x Z_{v-t}^{r}$ from (3.4) above with $\mu_{c}$ replaced by $r$ and $T-t$ replaced by $v-t$

$$
f(v-t, x, y)=\frac{1}{\sigma y \sqrt{v-t}} \varphi\left(\frac{1}{\sigma \sqrt{v-t}}\left[\log (y / x)-\left(r-\sigma^{2} / 2\right)(v-t)\right]\right)
$$

for $y>0$ where $\varphi$ is defined as in (3.15) above.

It is easy to verify by passing to the limit that

$$
\begin{aligned}
& J_{1}(t, x, T-, z)=\mu_{c} x \Phi\left(\frac{1}{\sigma \sqrt{T-t}}\left[\log \frac{z \wedge K}{x}-\left(r+\frac{\sigma^{2}}{2}\right)(T-t)\right]\right) \\
& J_{2}(t, x, T-, z)=0
\end{aligned}
$$

for $t \in[0, T), x>0$ and $z>0$. These expressions are useful in a computational treatment of the equations (4.7) and (4.8) below. The main result of this section may now be stated as follows.

Theorem 3. The arbitrage-free price of the British asset-or-nothing put option follows the early-exercise premium representation

$$
\begin{array}{ll}
V(t, x)=e^{-r(T-t)} G^{r}(t, x)+\int_{t}^{T} J_{1}\left(t, x, v, b_{1}(v)\right) d v & \text { if } \mu_{c}>r \\
V(t, x)=e^{-r(T-t)} G^{r}(t, x)+\int_{t}^{T} J_{2}\left(t, x, v, b_{2}(v)\right) d v & \text { if } \mu_{c}<0
\end{array}
$$

for all $(t, x) \in[0, T] \times(0, \infty)$, where the first term in each of (4.7) and (4.8) is the arbitragefree price of the European asset-or-nothing put option and the second term is the early-exercise premium. The rational exercise boundary of the British asset-or-nothing put option can be characterised as the unique continuous solutions $b_{1}:[0, T] \rightarrow \mathrm{R}_{+}$and $b_{2}:[0, T] \rightarrow \mathrm{R}_{+}$to the non-linear integral equations

$$
\begin{array}{ll}
F\left(t, b_{1}(t)\right)=\int_{t}^{T} J_{1}\left(t, b_{1}(t), v, b_{1}(v)\right) d v & \text { if } \mu_{c}>r \\
F\left(t, b_{2}(t)\right)=\int_{t}^{T} J_{2}\left(t, b_{2}(t), v, b_{2}(v)\right) d v & \text { if } \mu_{c}<0
\end{array}
$$

satisfying $0 \leq b_{1}(t) \leq h_{1}(t)$ and $b_{2}(t) \geq h_{2}(t)$ for all $t \in[0, T]$ where $h_{1}$ and $h_{2}$ are defined by (3.16) above.

Proof. We will first derive (4.7) and (4.8). Then we will show the uniqueness of (4.9) and (4.10).

1. Let $V:[0, T] \times(0, \infty) \rightarrow \mathrm{R}$ and $b:[0, T] \rightarrow \mathrm{R}_{+}$denote the unique solution to the freeboundary problem (3.23)-(3.27), set $C_{b}=\{(t, x) \in[0, T) \times(0, \infty) \mid(t, x) \in C\}$ and $D_{b}=\{(t, x) \in$ $[0, T) \times(0, \infty) \mid(t, x) \in D\}$, and let $\mathbb{L}_{X} V(t, x)=r x V_{x}(t, x)+\frac{\sigma^{2}}{2} x^{2} V_{x x}(t, x)$ for $(t, x) \in C_{b} \cup D_{b}$.

We summarise that $V$ and $b$ are continuous functions satisfying the following conditions

$$
V \text { is } C^{1,2} \text { on } C_{b} \cup D_{b}
$$




$$
\begin{aligned}
& b \text { is of bounded variation } \\
& \mathrm{P}\left(X_{t}=c\right)=0 \text { for all } t \in[0, T] \text { and } c>0 \\
& V_{t}+\mathbb{L}_{X} V-r V \text { is locally bounded on } C_{b} \cup D_{b} \\
& t \rightarrow V_{x}(t, b(t) \pm)=G_{x}^{\mu_{c}}(t, b(t)) \text { is continuous on }[0, T] .
\end{aligned}
$$

From these conditions we see that the local time-space formula is applicable to $(s, y) \rightarrow$ $e^{-r s} V(t+s, x y)$ with $t \in[0, T)$ and $x>0$ given and fixed (Peskir, 2005). This leads to

$$
\begin{aligned}
& e^{-r s} V\left(t+s, x X_{s}\right)=V(t, x) \\
& \quad+\int_{0}^{s} e^{-r v}\left(V_{t}+\mathbb{L}_{X} V-r V\right)\left(t+v, x X_{v}\right) \mathrm{I}\left(x X_{v} \neq b(t+v)\right) d v+M_{s}^{b} \\
& \quad+\frac{1}{2} \int_{0}^{s} e^{-r v}\left(V_{x}\left(t+v, x X_{v^{+}}\right)-V_{x}\left(t+v, x X_{v^{-}}\right)\right) \mathrm{I}\left(x X_{v}=b(t+v)\right) d \ell_{v}^{b}\left(X^{x}\right)
\end{aligned}
$$

where $M_{s}^{b}=\sigma \int_{0}^{s} e^{-r v} x X_{v} V_{x}\left(t+v, x X_{v}\right) d B_{v}$ is a continuous local martingale for $s \in[0, T-t]$ and $\ell^{b}\left(X^{x}\right)=\left(\ell_{v}^{b}\left(X^{x}\right)\right)_{0 \leq v \leq s}$ is the local time of $X^{x}=\left(x X_{v}\right)_{0 \leq v \leq s}$ on the curve $b$ for $s \in[0, T-t]$. Furthermore, since $V$ satisfies (3.23) on $C_{b}$ and equals $G^{\mu_{c}}$ on $D_{b}$, and the smooth-fit condition holds at $b$, we see that (4.16) implies

$$
\begin{aligned}
& e^{-r s} V\left(t+s, x X_{s}\right)=V(t, x) \\
& \quad+\int_{0}^{s} e^{-r v}\left(V_{t}+\mathbb{L}_{X} V-r V\right)\left(t+v, x X_{v}\right) \mathrm{I}\left(x X_{v} \in D\right) d v+M_{s}^{b}
\end{aligned}
$$

for $s \in[0, T-t]$ and $(t, x) \in[0, T) \times(0, \infty)$.

2. Since $M_{s}^{b}$ is a continuous local martingale, there exist a sequence of stopping times $\tau_{n}$ such that $\tau_{n} \uparrow \infty$ as $n \uparrow \infty$ and the stopped process $M_{s \wedge \tau_{n}}$ is a martingale. Replacing $s$ by $s \wedge \tau_{n}$ in (4.17), we get

$$
\begin{aligned}
& e^{-r\left(s \wedge \tau_{n}\right)} V\left(t+s \wedge \tau_{n}, x X_{s \wedge \tau_{n}}\right)=V(t, x) \\
& \quad+\int_{0}^{s \wedge \tau_{n}} e^{-r v}\left(V_{t}+\mathbb{L}_{X} V-r V\right)\left(t+v, x X_{v}\right) \mathrm{I}\left(x X_{v} \in D\right) d v \\
& \quad+M_{s \wedge \tau_{n}}^{b} .
\end{aligned}
$$

The martingale term vanishes when taking $\mathrm{E}$ on both sides. It follows

$$
\begin{aligned}
\mathrm{E}\left[e^{-r\left(s \wedge \tau_{n}\right)} V\left(t+s \wedge \tau_{n}, x X_{s \wedge \tau_{n}}\right)\right]=V(t, x) \\
+\mathrm{E}\left[\int_{0}^{s \wedge \tau_{n}} e^{-r v} H^{\mu_{c}}\left(t+v, x X_{v}\right) \mathrm{I}\left(x X_{v} \in D\right) d v\right]
\end{aligned}
$$

where $H^{\mu_{c}}(t, x)$ is well defined in (3.15). When taking $\lim _{n \uparrow \infty}$, it reduces to

$$
\begin{aligned}
& \mathrm{E}\left[e^{-r s} V\left(t+s, x X_{s}\right)\right]=V(t, x) \\
& \quad+\mathrm{E}\left[\int_{0}^{s} e^{-r v} H^{\mu_{c}}\left(t+v, x X_{v}\right) \mathrm{I}\left(x X_{v} \in D\right) d v\right] .
\end{aligned}
$$


3. Replacing $s$ by $T-t$ in (4.20), using that $V(T, x)=G^{\mu_{c}}(T, x)=x \mathrm{I}(x \leq K)$ for $x>0$ and from the optimal sampling theorem, we get

$$
\begin{aligned}
& e^{-r(T-t)} \mathrm{E}\left(x X_{T-t} \mathrm{I}\left(x X_{T-t}<K\right)\right) \\
& =V(t, x)+\int_{0}^{T-t} e^{-r v} \mathrm{E}\left[H^{\mu_{c}}\left(t+v, x X_{v}\right) \mathrm{I}\left(x X_{v} \in D\right)\right] d v \\
& =V(t, x)-\int_{t}^{T} J_{1}(t, x, v, b(v)) d v .
\end{aligned}
$$

Note that the left-hand side of (4.21) is $e^{-r(T-t)} G^{r}(t, x)$ and we will get the expression (4.7). It follows by (4.9) since $V(t, b(t))=G^{\mu_{c}}(t, b(t))$ for all $t \in[0, T]$. Similarly, when $\mu_{c}<0$, using (3.30) and (4.3) we see that (4.21) yields the representation (4.8). Furthermore, from (4.8) we see $b_{2}(t)$ solves (4.10). Next, we will turn to the uniqueness of $b$.

4. We will show that the rational exercise boundary is the unique solutions to (4.9) and (4.10) in the class of continuous functions $t \mapsto b_{1}(t)$ and $t \mapsto b_{2}(t)$ on $[0, T]$ satisfying $0 \leq$ $b_{1}(t) \leq h_{1}(t)$ and $b_{2}(t) \geq h_{2}(t)$ for all $t \in[0, T]$.

To prove this, we assume two continuous functions $c_{1}:[0, T] \rightarrow \mathrm{R}$ and $c_{2}:[0, T] \rightarrow \mathrm{R}$ which solves (4.9) and (4.10) and satisfies $0 \leq c_{1}(t) \leq h(t)$ and $c_{2}(t) \geq h_{2}(t)$ for all $t \in[0, T]$. From $(4.21)$, the function $U^{c}:[0, T] \times(0, \infty) \rightarrow \mathrm{R}$ is defined by

$$
\begin{aligned}
U^{c}(t, x)= & e^{-r(T-t)} \mathrm{E}\left[G^{\mu_{c}}\left(t, x X_{T-t}\right)\right] \\
& -\int_{0}^{T-t} e^{-r v} \mathrm{E}\left[H^{\mu_{c}}\left(t+v, x X_{v}\right) \mathrm{I}\left(x X_{v} \in D_{c}\right)\right] d v
\end{aligned}
$$

where

$$
D_{c}=\left\{(t, x) \in[0, T] \times(0, \infty) \mid U^{c}(t, x)=G^{\mu_{c}}(t, x)\right\} .
$$

It is easy to know that if $c_{1}$ and $c_{2}$ solves (4.9) and (4.10) then $U^{c}\left(t, c_{i}(t)\right)=G^{\mu_{c}}\left(t, c_{i}(t)\right)$ for all $t \in[0, T]$ and $i=1,2$. So $D_{c}$ plays the role of the stopping region for $c_{1}$ and $c_{2}$.

(i) First we will show that $U^{c}(t, x)=G^{\mu_{c}}(t, x)$ for all $(t, x) \in[0, T] \times(0, \infty)$ such that $(t, x) \in D_{c}$. Take such $(t, x)$ and the Markov property of $X$ implies

$$
e^{-r s} U^{c}\left(t+s, x X_{s}\right)-\int_{0}^{s} e^{-r v} H^{\mu_{c}}\left(t+v, x X_{v}\right) \mathrm{I}\left(x X_{v} \in D_{c}\right) d v
$$

is a continuous martingale under $\mathrm{P}$ for $s \in[0, T-t]$. Consider the stopping time

$$
\sigma_{c}=\inf \left\{s \in[0, T-t] \mid x X_{x} \notin D_{c}\right\}
$$

under P. We see that

$$
U^{c}\left(t+\sigma_{c}, x X_{\sigma_{c}}\right)=G^{\mu_{c}}\left(t+\sigma_{c}, x X_{\sigma_{c}}\right)
$$

since $U^{c}\left(t, c_{i}(t)\right)=G^{\mu_{c}}\left(t, c_{i}(t)\right)$ for all $t \in[0, T]$ and $i=1,2$ and $U^{c}(T, x)=G^{\mu_{c}}(T, x)$ for all $x>0$. Now we replace $s$ by $\sigma_{c}$ in (4.24) and take $\mathrm{E}$ on both sides of (4.26), from the optional 


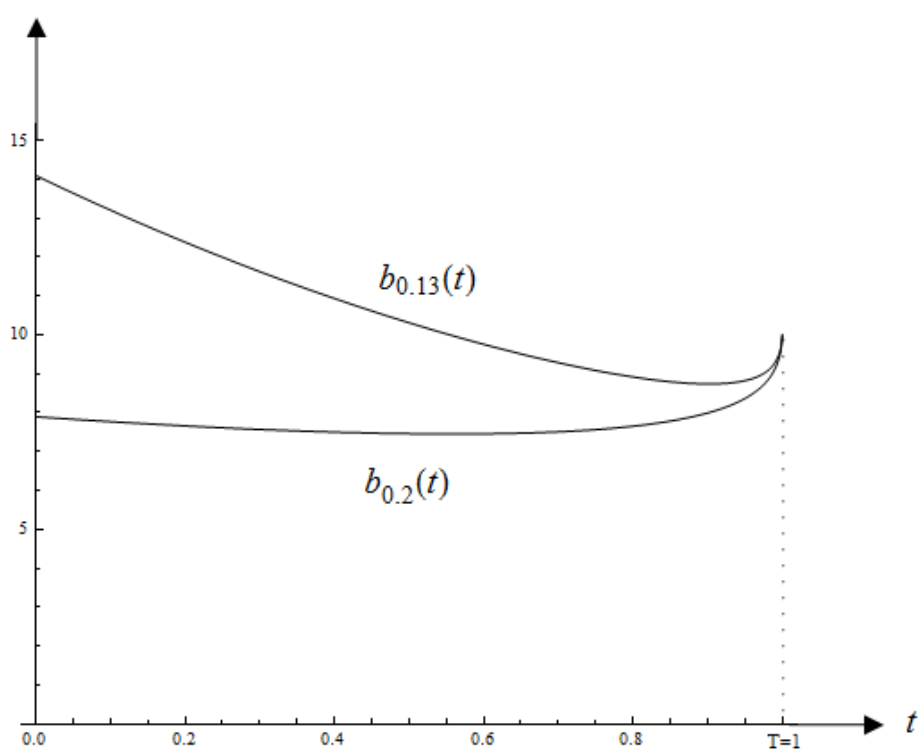

Figure 3: A computer drawing comparing the rational exercise boundary of the British asset-or-nothing put option with $K=10, T=1, r=0.1, \sigma=0.4$ when the contract drift $\mu_{c}=0.13$ and $\mu_{c}=0.2$.

sampling theorem

$$
\begin{aligned}
U^{c}(t, x) & =\mathrm{E}\left[e^{-r \sigma_{c}} U^{c}\left(t+\sigma_{c}, x X_{\sigma_{c}}\right)\right]-\mathrm{E}\left[\int_{0}^{\sigma_{c}} e^{-r v} H^{\mu_{c}}\left(t+v, x X_{v}\right) \mathrm{I}\left(x X_{v} \in D_{c}\right) d v\right] \\
& =\mathrm{E}\left[e^{-r \sigma_{c}} G^{\mu_{c}}\left(t+\sigma_{c}, x X_{\sigma_{c}}\right)\right]-\mathrm{E}\left[\int_{0}^{\sigma_{c}} e^{-r v} H^{\mu_{c}}\left(t+v, x X_{v}\right) d v\right] \\
& =G^{\mu_{c}}(t, x)
\end{aligned}
$$

where we use (3.12) in the last step.

(ii) Next, we will show that $U^{c}(t, x) \leq V(t, x)$ for all $(t, x) \in[0, T] \times(0, \infty)$. Then we consider the stopping time

$$
\tau_{c}=\inf \left\{s \in[0, T-t] \mid x X_{s} \in D_{c}\right\}
$$

under P.

If $(t, x) \in D_{c}$ then $\tau_{c}=0$ so that $U^{c}(t, x)=G^{\mu_{c}}(t, x)$ from (i) above. On the other hand, if $(t, x) \notin D_{c}$ then $U^{c}\left(t, c_{i}(t)\right)=G^{\mu_{c}}\left(t, c_{i}(t)\right)$ for $i=1,2$ and $U^{c}(T, x)=G^{\mu_{c}}(T, x)$ for all $x>0$. From above analysis, we claim that

$$
U^{c}\left(t+\tau_{c}, x X_{\tau_{c}}\right)=G^{\mu_{c}}\left(t+\tau_{c}, x X_{\tau_{c}}\right) .
$$

Replacing $s$ by $\tau_{c}$ in (4.24) and taking $\mathrm{E}$ on both sides, from the optional sampling theorem we get

$$
\begin{aligned}
U^{c}(t, x) & =\mathrm{E}\left[e^{-r \tau_{c}} U^{c}\left(t+\tau_{c}, x X_{\tau_{c}}\right)\right]-\mathrm{E}\left[\int_{0}^{\tau_{c}} e^{-r v} H^{\mu_{c}}\left(t+v, x X_{v}\right) \mathrm{I}\left(x X_{v}(t, x) \in D_{c}\right) d v\right] \\
& =\mathrm{E}\left[e^{-r \tau_{c}} G^{\mu_{c}}\left(t+\tau_{c}, x X_{\tau_{c}}\right)\right] \leq V(t, x)
\end{aligned}
$$




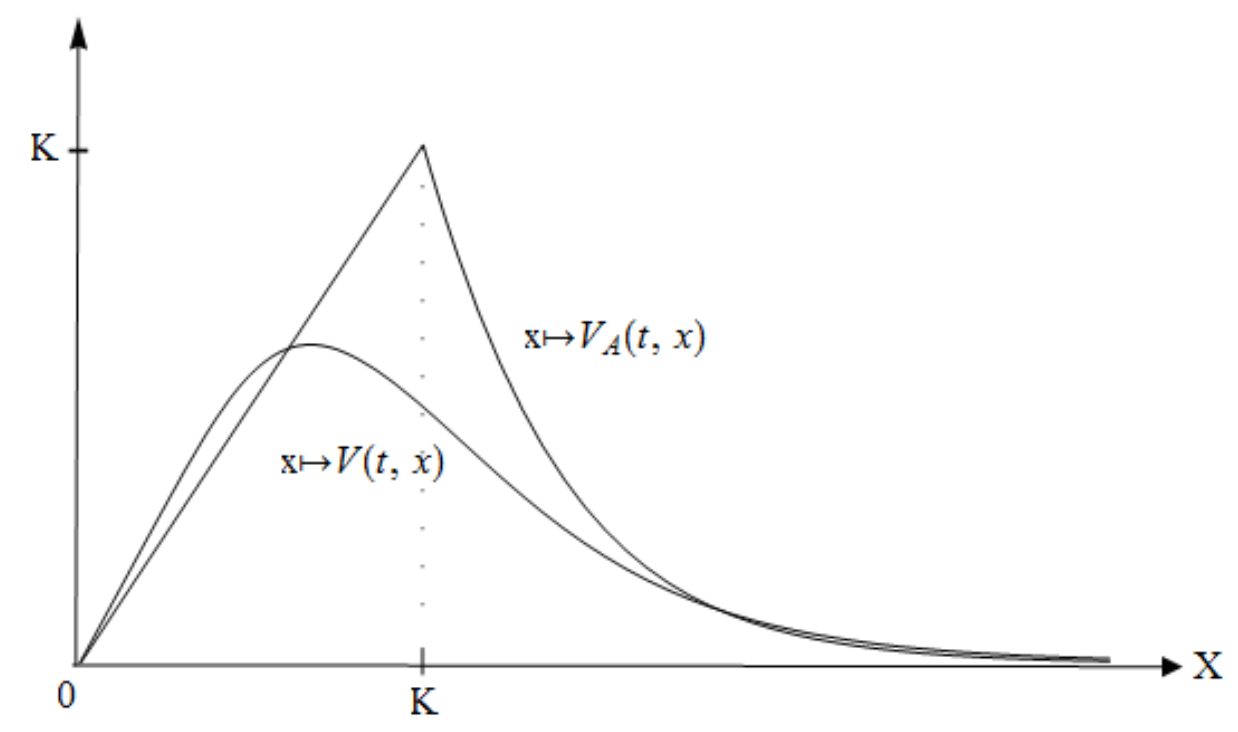

Figure 4: A computer comparison of the value of the British/American asset-or-nothing put options for $t$ given and fixed.

where

$$
V(t, x)=\sup _{0 \leq \tau \leq T-t} \mathrm{E}_{t, x}\left[e^{-r \tau} G^{\mu_{c}}\left(t+\tau, x X_{\tau}\right)\right]
$$

Therefore, $U^{c}(t, x) \leq V(t, x)$ as claimed.

(iii) Next, we are going to show that $D_{c} \subseteq D_{b}$ for all $t \in[0, T]$ (where $D_{b}$ is the original stopping set from problem (3.8)). It is equivalent to that $b_{1}(t) \leq c_{1}(t)$ and $b_{2}(t) \geq c_{2}(t)$. We will prove in contradiction. Suppose that there exists $t \in[0, T]$ such that $c_{1}(t)<b_{1}(t)$ and $c_{2}(t)>b_{2}(t)$. Take any $x \leq c_{1}(t)$ or $x \geq c_{2}(t)$ and consider the stopping time

$$
\sigma_{b}=\inf \left\{s \in[0, T-t] \mid x X_{s} \notin D_{b}\right\}
$$

under P. Replacing $s$ with $\sigma_{b}$ in (4.17), taking $\mathrm{E}$ on both sides and applying the optional sampling theorem we get

$$
\mathrm{E}\left[e^{-r \sigma_{b}} V\left(t+\sigma_{b}, x X_{\sigma_{b}}\right)\right]=V(t, x)+\mathrm{E}\left[\int_{0}^{\sigma_{b}} e^{-r v} H^{\mu_{c}}\left(t+v, x X_{v}\right) d v\right] .
$$

Repeating the above process in (4.24), we find

$$
\mathrm{E}\left[e^{-r \sigma_{b}} U^{c}\left(t+\sigma_{b}, x X_{\sigma_{b}}\right)\right]=U^{c}(t, x)+\mathrm{E}\left[\int_{0}^{\sigma_{b}} e^{-r v} H^{\mu_{c}}\left(t+v, x X_{v}\right) \mathrm{I}\left(x X_{v} \in D_{c}\right) d v\right] .
$$

We see $U^{c}(t, x)=G^{\mu_{c}}(t, x)=V(t, x)$ since $x \leq c_{1}(t)$ or $x \geq c_{2}(t)$ and by (ii) we know $U^{c}\left(t+\sigma_{b}, x X_{\sigma_{b}}\right) \leq V\left(t+\sigma_{b}, x X_{\sigma_{b}}\right)$, then (4.33) and (4.34) imply that

$$
\mathrm{E}\left[\int_{0}^{\sigma_{b}} e^{-r v} H^{\mu_{c}}\left(t+v, x X_{v}\right) \mathrm{I}\left(x X_{v} \notin D_{c}\right) d v\right] \geq 0
$$


On the other hand, the fact that $b_{1}$ lies below $h_{1}$ and $b_{2}$ lies above $h_{2}$ forces (4.35) to be strictly negative and provides a contradiction. Therefore, $D_{c} \subseteq D_{b}$ as claimed.

(iv) Next, we will show that $D_{c}=D_{b}$ for all $t \in[0, T]$. We first assume the reverse case that there exists $t \in[0, T]$ such that $c_{1}(t)>b_{1}(t)$ or $c_{2}(t)<b_{2}(t)$. Take any $x \in\left(b_{1}(t), c_{1}(t)\right)$ or $x \in\left(c_{2}(t), b_{2}(t)\right)$ and consider the stopping time

$$
\tau_{b}=\inf \left\{s \in[0, T-t] \mid x X_{s} \in D_{b}\right\}
$$

under P. Replacing $s$ with $\tau_{b}$ in (4.17) and (4.24), taking $\mathrm{E}$ on both sides and applying the optional sampling theorem we get

$$
\begin{aligned}
& \mathrm{E}\left[e^{-r \tau_{b}} V\left(t+\tau_{b}, x X_{\tau_{b}}\right)\right]=V(t, x) \\
& \mathrm{E}\left[e^{-r \tau_{b}} U^{c}\left(t+\tau_{b}, x X_{\tau_{b}}\right)\right]=U^{c}(t, x)+\mathrm{E}\left[\int_{0}^{\tau_{b}} e^{-r v} H^{\mu_{c}}\left(t+v, x X_{v}\right) \mathrm{I}\left(x X_{v} \in D_{c}\right) d v\right] .
\end{aligned}
$$

We see that $U^{c}\left(t+\tau_{b}, x X_{\tau_{b}}\right)=G^{\mu_{c}}\left(t+\tau_{b}, x X_{\tau_{b}}\right)=V\left(t+\tau_{b}, x X_{\tau_{b}}\right)$ by (i) and (iii) above since $b_{1} \leq c_{1}$ and $c_{2} \leq b_{2}$. Furthermore, by (ii) we know $U^{c} \leq V$ so (4.37) and (4.38) lead to

$$
\mathrm{E}\left[\int_{0}^{\tau_{b}} e^{-r v} H^{\mu_{c}}\left(t+v, x X_{v}\right) \mathrm{I}\left(x X_{v} \in D_{c}\right) d v\right] \geq 0 .
$$

However, the fact that $c_{1}$ lies below $h_{1}$ and $c_{2}$ above $h_{2}$ forces (4.39) to be strictly negative and provides a contradiction. Therefore, $D_{c}=D_{b}$ as claimed.

\section{$5 \quad$ Financial analysis of the British asset-or-nothing put option}

In this section, we focus on the practical features of the British asset-or-nothing put option. We compare it with the American and European asset-or-nothing put option that is universally traded. We address the problem as to what the return would be if the price of the underlying asset enters the given region at a given time. We use 'skeletal' approach of Peskir and Samee (2011), with a similar parameter set so that we can make reference to the results by Peskir and Samee (2011).

1. As shown in Figures 1 and 2, the rational exercise strategy of the British asset-ornothing put option varies with different contract drift $\mu_{c}$. Where depending on the value of $\mu_{c}$, the boundary $b$ is either an increasing function of time, a skewed U-shaped function of time, or an intermediate case where $b$ can take either of the two shapes depending on the size of $T$.

For $\mu_{c}>r$, we witness the same three regimes for the optimal stopping boundary $b$ as Peskir and Samee (2011). In particular, as $\mu_{c}$ gets closer to $r$, we see that $b(0) \uparrow \infty$ making the stock price always below $b(0)$. It would be optimal to stop immediately, making the buyer overprotected. On the other hand, when $\mu_{c} \uparrow \infty$, it is not optional to exercise the option before time $T$, reducing the British asset-or-nothing put option to the European asset-or-nothing put option. Therefore, the contract drift $\mu_{c}$ should not be too close to $r$ (since in this case the buyer is overprotected) and should not be too large (since in this case the British asset-or-nothing put option effectively reduces to the European asset-or-nothing put option). 


\begin{tabular}{|l|c|c|c|c|c|c|c|}
\hline Time $($ months $)$ & 0 & 2 & 4 & 6 & 8 & 10 & 12 \\
\hline Exercise at $\mathrm{K}$ with $\mu_{c}=0.13$ & $101 \%$ & $100 \%$ & $99 \%$ & $98 \%$ & $97 \%$ & $97 \%$ & $198 \%$ \\
\hline Exercise at $\mathrm{K}$ with $\mu_{c}=0.20$ & $95 \%$ & $94 \%$ & $93 \%$ & $93 \%$ & $94 \%$ & $95 \%$ & $203 \%$ \\
\hline Exercise at 11 with $\mu_{c}=0.13$ & $89 \%$ & $86 \%$ & $82 \%$ & $78 \%$ & $71 \%$ & $58 \%$ & $0 \%$ \\
\hline Exercise at 11 with $\mu_{c}=0.20$ & $80 \%$ & $78 \%$ & $75 \%$ & $72 \%$ & $67 \%$ & $55 \%$ & $0 \%$ \\
\hline Exercise at 12 with $\mu_{c}=0.13$ & $76 \%$ & $71 \%$ & $66 \%$ & $59 \%$ & $48 \%$ & $29 \%$ & $0 \%$ \\
\hline Exercise at 12 with $\mu_{c}=0.20$ & $67 \%$ & $63 \%$ & $59 \%$ & $53 \%$ & $44 \%$ & $27 \%$ & $0 \%$ \\
\hline Exercise at 13 with $\mu_{c}=0.13$ & $64 \%$ & $58 \%$ & $51 \%$ & $42 \%$ & $30 \%$ & $13 \%$ & $0 \%$ \\
\hline Exercise at 13 with $\mu_{c}=0.20$ & $55 \%$ & $50 \%$ & $44 \%$ & $37 \%$ & $27 \%$ & $11 \%$ & $0 \%$ \\
\hline Exercise at 14 with $\mu_{c}=0.13$ & $53 \%$ & $46 \%$ & $39 \%$ & $30 \%$ & $18 \%$ & $5 \%$ & $0 \%$ \\
\hline Exercise at 14 with $\mu_{c}=0.20$ & $44 \%$ & $39 \%$ & $33 \%$ & $25 \%$ & $16 \%$ & $4 \%$ & $0 \%$ \\
\hline Exercise at 15 with $\mu_{c}=0.13$ & $43 \%$ & $36 \%$ & $29 \%$ & $20 \%$ & $10 \%$ & $2 \%$ & $0 \%$ \\
\hline Exercise at 15 with $\mu_{c}=0.20$ & $35 \%$ & $30 \%$ & $24 \%$ & $17 \%$ & $9 \%$ & $1 \%$ & $0 \%$ \\
\hline
\end{tabular}

Table 1: Returns observed upon exercising the British asset-or-nothing put options (with $\mu_{c}=0.13$ and $\left.\mu_{c}=0.2\right)$ above the strike price $K$. The returns are calculated by $R(t, x)=$ $G^{\mu_{c}}(t, x) / V(0,11)$. The parameter set is same as in Figure 3 i.e. $K=10, T=1, r=0.1$, $\sigma=0.4$ and the initial stock price is 11 .

2. In the numerical example below (see Tables 1,2 and 3), the parameter values have been chosen to present the practical features of the British asset-or-nothing put option. We assume that the initial stock price is 11 , the strike price $K=10$, the maturity time $T=1$, the interest rate $r=0.1$ and volatility coefficient $\sigma=0.4$. By considering the set of parameters above, the arbitrage-free price of the British asset-or-nothing put option using Equation (4.7) is 4.43519 with $\mu_{c}=0.13$ and 4.3368 with $\mu_{c}=0.2$. The price of the European asset-or-nothing put option is 2.70204 that can be evaluated using the formula in Hull (2010). We exploit the same method to find the price of the American asset-or-nothing put option $V_{A}(0,11)=7.88469$. A direct comparison for the values of the British and American asset-or-nothing put options is shown in Figure 4. In terms of the price sizes it can be seen that this example is quite typical since the price of the British asset-or-nothing put option lies between the prices of the European option and the American option. We get that the closer the contract drift gets to $r$, the protection feature is stronger and the option price is more expensive as stated above. If the contract drift $\mu_{c}$ is too close to $r$, the protection feature works and makes the price of the British option extremely close to the American option. If $\mu_{c} \uparrow \infty$ the British asset-or-nothing put option will be reduced to the European asset-or-nothing put option. On the other hand, if the initial stock price is 3, the European asset-or-nothing put option is 2.9843 and the American assetor-nothing put option is 3 . The British asset-or-nothing put option is 2.99448 with $\mu_{c}=-0.1$ that is also between the prices of the European and American versions.

3. Table 1 illustrates the power of the protection feature in practice. For instance, if the stock price is 11 halfway to maturity then the option holder can exercise to a payoff with a reimbursement of $72 \%-78 \%$ of the initial investment. However, in the case of the American asset-or-nothing put option, the holder is out-of-the-money and would receive zero payoff. We see that the size of the reimbursement also varies with the contract drift as analysed in 


\begin{tabular}{|l|c|c|c|c|c|c|c|}
\hline Time(months) & 0 & 2 & 4 & 6 & 8 & 10 & 12 \\
\hline Exercise at K (British) & $118 \%$ & $117 \%$ & $116 \%$ & $114 \%$ & $113 \%$ & $113 \%$ & $231 \%$ \\
\hline Exercise at K (American) & $127 \%$ & $127 \%$ & $127 \%$ & $127 \%$ & $127 \%$ & $127 \%$ & $127 \%$ \\
\hline Exercise at b (British) & $141 \%$ & $144 \%$ & $147 \%$ & $152 \%$ & $159 \%$ & $171 \%$ & $231 \%$ \\
\hline Exercise at b (American) & $100 \%$ & $98 \%$ & $96 \%$ & $95 \%$ & $95 \%$ & $98 \%$ & $127 \%$ \\
\hline Exercise at 8 (British) & $140 \%$ & $142 \%$ & $145 \%$ & $149 \%$ & $157 \%$ & $171 \%$ & $184 \%$ \\
\hline Exercise at 8 (American) & $101 \%$ & $101 \%$ & $101 \%$ & $101 \%$ & $101 \%$ & $101 \%$ & $101 \%$ \\
\hline Exercise at 6 (British) & $138 \%$ & $139 \%$ & $140 \%$ & $141 \%$ & $142 \%$ & $141 \%$ & $138 \%$ \\
\hline Exercise at 6 (American) & $76 \%$ & $76 \%$ & $76 \%$ & $76 \%$ & $76 \%$ & $76 \%$ & $76 \%$ \\
\hline Exercise at 4 (British) & $103 \%$ & $102 \%$ & $100 \%$ & $98 \%$ & $96 \%$ & $94 \%$ & $92 \%$ \\
\hline Exercise at 4 (American) & $51 \%$ & $51 \%$ & $51 \%$ & $51 \%$ & $51 \%$ & $51 \%$ & $51 \%$ \\
\hline Exercise at 2 (British) & $53 \%$ & $51 \%$ & $50 \%$ & $49 \%$ & $48 \%$ & $47 \%$ & $46 \%$ \\
\hline Exercise at 2 (American) & $25 \%$ & $25 \%$ & $25 \%$ & $25 \%$ & $25 \%$ & $25 \%$ & $25 \%$ \\
\hline
\end{tabular}

Table 2: Returns observed upon exercising the British asset-or-nothing put options (with $\left.\mu_{c}=0.2\right)$ at and below the strike price $K$. The returns are calculated by $R(t, x)=$ $G^{\mu_{c}}(t, x) / V(0,11)$ and $R_{A}(t, x)=x / V_{A}(0,11)$ respectively. The parameter set is same as in Figure 3 i.e. $K=10, T=1, r=0.1, \sigma=0.4$ and the initial stock price is 11.

the former paragraph. When the contract drift gets closer to $r$, the option holder gets more protection as well as greater reimbursement.

4. In Table 2, we compare the returns of the British asset-or-nothing put option and the American version option as the stock price is moving favourably. The result generally indicates that the British option outperforms the American option except a few points. In Table 3, we compare the protection feature of the British option with the reimbursement of American option if the holder of the latter can choose to sell his option freely without friction. The value of the American asset-or-nothing put option is always 1 below the strike price $K$ so we make the region above $K$. Actually, the payoff of the American option above $K$ is 0 and when this happens we assume that the option holder can sell his option. We see in Table 3 that this makes the returns very close to the British asset-or-nothing put option. However, in the real market the option holder's ability to sell the option is affected by many exogenous factors such as the friction costs, taxes, the liquidity of the market and so on. Therefore, from this point of view it makes the British style option more attractive since the protection feature to the British option is intrinsic and endogenous.

\section{Acknowledgement}

The author is grateful to Goran Peskir, Yerkin Kitapbayev and Shi Qiu for the informative discussions. 


\begin{tabular}{|l|c|c|c|c|c|c|c|}
\hline Time(months) & 0 & 2 & 4 & 6 & 8 & 10 & 12 \\
\hline Exercise at K (British) & $118 \%$ & $117 \%$ & $116 \%$ & $114 \%$ & $113 \%$ & $113 \%$ & $231 \%$ \\
\hline Selling at K (American) & $127 \%$ & $127 \%$ & $127 \%$ & $127 \%$ & $127 \%$ & $127 \%$ & $127 \%$ \\
\hline Exercise at 11 (British) & $103 \%$ & $100 \%$ & $96 \%$ & $91 \%$ & $83 \%$ & $68 \%$ & $0 \%$ \\
\hline Selling at 11 (American) & $100 \%$ & $98 \%$ & $95 \%$ & $91 \%$ & $84 \%$ & $70 \%$ & $0 \%$ \\
\hline Exercise at 12 (British) & $88 \%$ & $83 \%$ & $77 \%$ & $68 \%$ & $56 \%$ & $34 \%$ & $0 \%$ \\
\hline Selling at 12 (American) & $78 \%$ & $75 \%$ & $70 \%$ & $63 \%$ & $52 \%$ & $32 \%$ & $0 \%$ \\
\hline Exercise at 13 (British) & $74 \%$ & $68 \%$ & $60 \%$ & $50 \%$ & $36 \%$ & $15 \%$ & $0 \%$ \\
\hline Selling at 13 (American) & $60 \%$ & $56 \%$ & $50 \%$ & $42 \%$ & $31 \%$ & $13 \%$ & $0 \%$ \\
\hline Exercise at 14 (British) & $61 \%$ & $54 \%$ & $45 \%$ & $35 \%$ & $21 \%$ & $6 \%$ & $0 \%$ \\
\hline Selling at 14 (American) & $46 \%$ & $42 \%$ & $36 \%$ & $28 \%$ & $17 \%$ & $5 \%$ & $0 \%$ \\
\hline Exercise at 15 (British) & $50 \%$ & $43 \%$ & $34 \%$ & $24 \%$ & $12 \%$ & $2 \%$ & $0 \%$ \\
\hline Selling at 15 (American) & $36 \%$ & $31 \%$ & $25 \%$ & $18 \%$ & $9 \%$ & $2 \%$ & $0 \%$ \\
\hline
\end{tabular}

Table 3: Returns observed upon exercising the British asset-or-nothing put options (with $\mu_{c}=0.2$ ) above the strike price $K$ compared with returns received upon selling the American asset-or-nothing put option in the same contingency. The returns are calculated by $R_{B}(t, x)=G^{\mu_{c}}(t, x) / V(0,11)$ and $R_{A}(t, x)=V_{A}(t, x) / V_{A}(0,11)$. The parameter set is same as in Figure 3 i.e. $K=10, T=1, r=0.1, \sigma=0.4$ and the initial stock price is 11 .

\section{References}

Al-Fagih, L. (2014). The British knock-in put option. Research Report No. 5, Probab. Statist. Group Manchester.

Al-Fagih, L. (2015). The british knock-out put option. International Journal of Theoretical and Applied Finance, 18(02):1550008.

Black, F. and Scholes, M. (1973). The pricing of options and corporate liabilities. The Journal of Political Economy, 81(3):637-654.

Du Toit, J. and Peskir, G. (2007). The trap of complacency in predicting the maximum. The Annals of Probability, pages 340-365.

Geske, R. (1979). The valuation of compound options. Journal of Financial Economics, 7(1):6381.

Glover, K., Peskir, G., and Samee, F. (2010). The British Asian option. Sequential Analysis, $29(3): 311-327$.

Glover, K., Peskir, G., and Samee, F. (2011). The British Russian option. Stochastics An International Journal of Probability and Stochastic Processes, 83(4-6):315-332.

Gukhal, C. R. (2004). The compound option approach to American options on jump-diffusions. Journal of Economic Dynamics and Control, 28(10):2055-2074.

Hull, J. (2010). Options, futures, and other derivatives. Pearson Education India. 
Kitapbayev, Y. (2015). The British lookback option with fixed strike. Applied Mathematical Finance, 22(3):238-260.

Merton, R. C. (1973). Theory of rational option pricing. The Bell Journal of Economics and Management Science, 4(1):141-183.

Peskir, G. (2005). A change-of-variable formula with local time on curves. Journal of Theoretical Probability, 18(3):499-535.

Peskir, G. and Samee, F. (2011). The British put option. Applied Mathematical Finance, 18(6):537-563.

Peskir, G. and Samee, F. (2013). The British call option. Quantitative Finance, 13(1):95-109.

Peskir, G. and Shiryaev, A. (2006). Optimal stopping and free-boundary problems. Birkhäuser Basel.

Shiryaev, A. N. and Kruzhilin, N. (1999). Essentials of stochastic finance: facts, models, theory. World Scientific Singapore.

\author{
Min Gao \\ School of Mathematics \\ The University of Manchester \\ Oxford Road \\ Manchester M13 9PL \\ United Kingdom \\ Min.Gao@manchester .ac.uk
}

